\title{
Case Report: Various Retinal Vascular Manifestations of Neurofibromatosis-1 in a Single Patient
}

\author{
Meisam Ghanbari ${ }^{12^{*}}$ (D), Mehrnoosh Maalhagh ${ }^{1,2}$ (D), Vahid Beigi ${ }^{1,2}$ (D) \\ 1. Poostchi Eye Research Center, Shiraz University of Medical Sciences, Shiraz, Iran. \\ 2. Department of Ophthalmology, School of Medicine, Shiraz University of Medical Sciences, Shiraz, Iran.
}

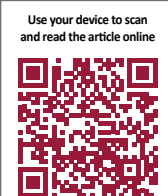

Citation Ghanbari M, Maalhagh M, Beigi V. Various Retinal Vascular Manifestations of Neurofibromatosis-1 in a Single Patient. JAMSAT. 2018; 4(1):27-30. https://doi.org/10.32598/jamsat.4.1.27

dol $\mathrm{https://doi.org/10.32598/jamsat.4.1.27}$

\section{(c) (i) (3)}

Article info:

Received: 13 Sep 2017

Accepted: 28 Dec 2017

Available Online: 01 Mar 2018

\section{Keywords:}

Neurofibromatosis type 1,

Retina, Choroid, Iris

\section{ABSTRACT}

Objectives: To report an Iranian woman with a variety of retinal vascular manifestations secondary to Neurofibromatosis -1 .

Case Report: A 49 years old woman known case of Neurofibromatosis-1 (NF-1) presented with decreased vision in her right eye. We found peculiar retinal and choroidal findings. Mild vascular tortuosity and few hard exudates were the prominent finding in right eye fundus examination; however, left eye showed inferior hemi retinal artery macro aneurysm. Fluorescein angiography, revealed multiple bright choroidal nodules in both eyes. Left eye findings were in favor of branch retinal artery occlusion and branch retinal vein occlusion.

Discussion: As vascular and microvascular manifestations of NF-1 are being reported recently, further investigational studies for retinal vascular associations in NF-1 patients appears reasonable. Complete ophthalmic examinations in each NF-1 patient seems essential to prevent potential threatening complications of vascular events in these patients.

\section{Highlights}

- Neurofibromatosis type 1 is an autosomal dominant phakomatous disorder.

- Neurofibromatosis type 1 can have various ocular manifestations such as neurofibromas of the eyelids, congenital glaucoma, and so on.

- Our case presented with typical iris nodules, retinal branch arterial occlusion as well as macroaneurysm, and multiple choroidal ovoid lesions in both eyes. 


\section{Plain Language Summary}

Neurofibromatosis type 1 is characterized with multiple neurofibromas presenting in various organs. This is a phakomatous disorder inherited in autosomal dominant pattern. Our patient was a typical case of neurofibromatosis type 1 . The presented patient had typical neurofibromas throughout her face and her diagnosis was confirmed by dermatologist. She complained of gradual decrease in visual acuity in both eyes. Our ocular examination revealed many ocular manifestations of this disease like typical Lisch nodules in iris in both eyes and other abnormalities. Fundus examination showed arterial filling defect in favor of branch retinal arterial occlusion with concomitant saccular dilatation compatible with arterial macroaneurysm. This case was a collection of ocular manifestations of type 1 neurofibromatosis in a single patient.

\section{Introduction}

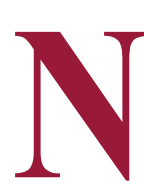

eurofibromatosis type 1 (NF-1), or von Recklinghausen disease is a phakomatous disorder [1]. The disease follows an autosomal dominant inheritance trait, which approximately affects as many as 1 in every 3000 newborns [2]. Ocular manifestations include Lisch nodules of the iris, optic gliomata and choroidal nodules which are implicated as diagnostic criteria, as well as neurofibromas of eyelids, congenital glaucoma, astrocytic hamartoma and microvascular retinal abnormalities [3-5], Multiple ovoid choroidal nodules are the classic choroidal findings in Neurofibromatosis known as "choroidal Neurofibromatosis" [5].

The disease has also established ischemic manifestations in systemic vasculature, primarily involving brain, renal and cerebral vasculature [6]. To date, novel

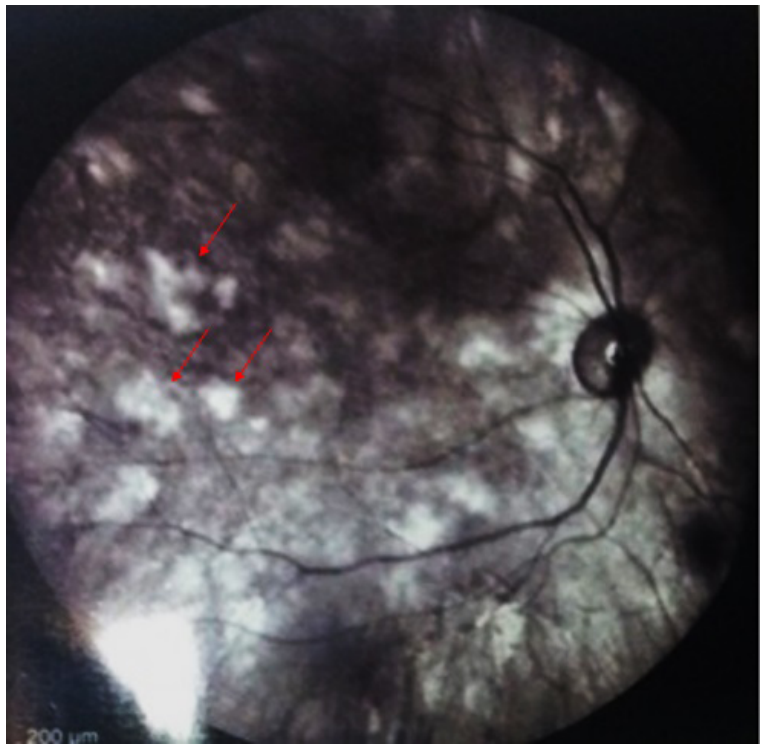

JAMSAT

Figure 1. Multiple skin-colored soft nodules (neurofibromas) findings among retinal vascular abnormalities include few cases of retinal vascular occlusions [7-9], peripheral retinal ischemia and corkscrew retinal venules [1012]. We describe a middle aged Iranian woman, case of NF-1 with the mentioned peculiar findings along with retinal arterial macroaneurysm.

\section{Case Report}

A 49 years old woman presented with a long history of decreased vision in her right eye worsening recently. Her Best Corrected Visual Acuity (BCVA) in right eye was 18.20 and the left eye was 6.20. General examination revealed multiple apparent neurofibromas throughout the face (Figure 1) as well as axillary freckles and café au lait spots. Slit lamp examination showed a clear cornea, normal anterior chamber, phakic lenses and Lisch nodules in both eyes.

On fundus examination of the right eye, mild vascular tortuosity and few hard exudates were the prominent finding; however, left eye showed temporal disc pallor, localized saccular dilatation of inferior hemiretinal artery at its 1 st bifurcation typical of arterial macroaneurysm, attenuation of the vessels and a distal embolus

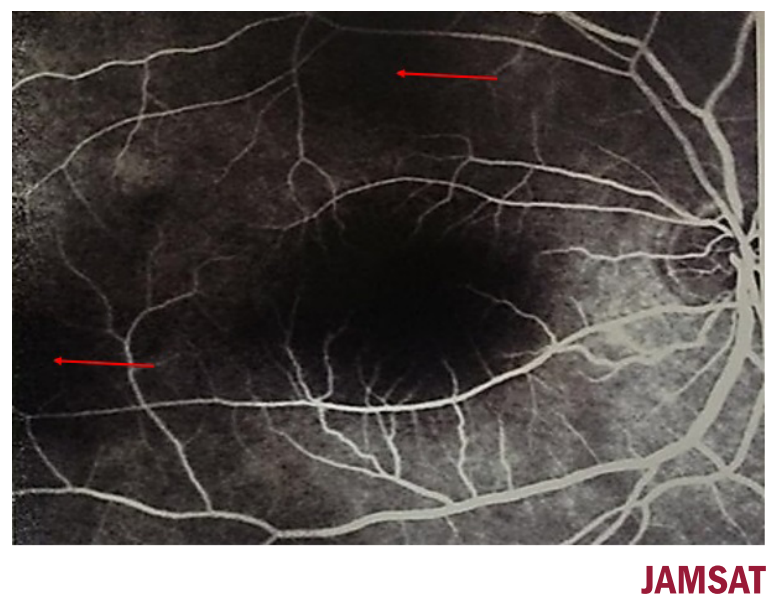

Figure 2. Infrared image showing patchy choroidal filling defects 


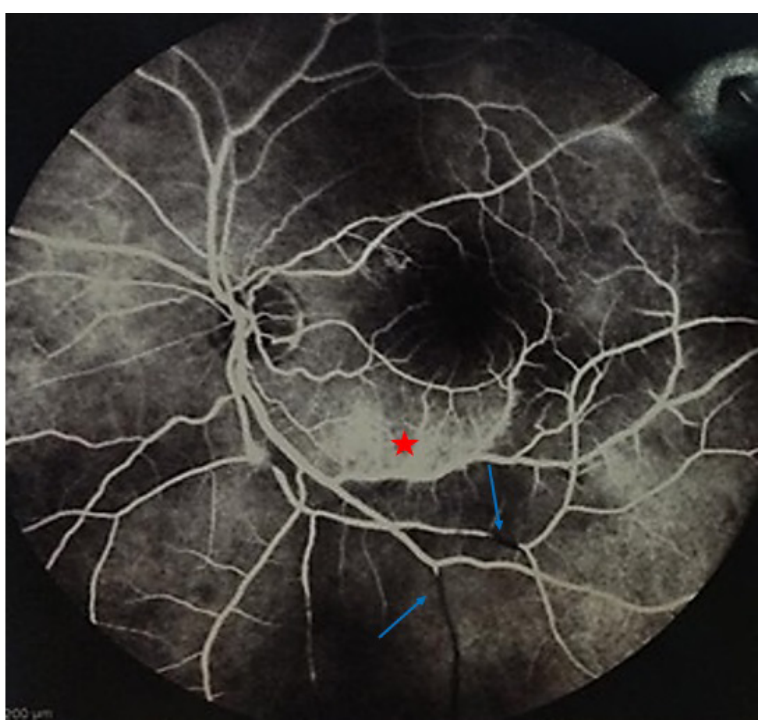

JAMSAT

Figure 3. Fluorescein angiography showing arterial and venous filling defect and aneurysm

Left: Fluorescein retinal angiography of the right eye showing multiple, patchy areas of ischemia (Red arrow); Right: Fluorescein retinal angiography of the left eye shows hyperfluoresence at the site of saccular dilation in inferotemporal retinal artery (Red asterisk) with successive filling defects in the same artery and its corresponding vein (Blue arrow)

occluding the successive branching. Furthermore, there was moderate venous dilatation and mild tortuosity in inferotemporal quadrant with venous sheathing. Infrared reflectance image of fundus revealed multiple bright and patchy choroidal leisons in both eyes (Figure 2).

Fluorescein angiography of left eye showed hyperfluorescence and leakage at the site of localized saccular dilatation and filling defects in both arteries and veins of inferotemporal quadrant may be in favor of Branch Retinal Artery Occlusion (BRAO) and Branch Retinal Vein Occlusion (BRVO), respectively but typical findings related to retinal and vein occlusion was not obvious in fluorescein angiography and not compatible with BRAO or BRVO. Multiple areas of capillary non perfusion areas were seen in both eyes (Figure 3).

\section{Discussion}

Neurofibromatosis-1 is a genetic phakomatosis disorder in which ophthalmologists frequently suggest, establish or confirm the diagnosis. Retinal findings known to be associated with NF-1 include retinal astrocytoma, capillary hemangioma, retinal hamartoma and microvascular retinal abnormalities [3, 4]. Nevertheless, Choroidal Neurofibromatosis, first described in postmortem studies is defined as oval multiple cho- roidal nodules is the classic choroidal finding in NF-1 patients [5]. There are reported rare cases of retinal vascular abnormalities as corkscrewing of parafoveal venules [10] and retinal vascular occlusive events in NF-1 patients [7-9].

In this presenting case the infrared reflectance image of fundus showed patchy hyper reflective choroidal alterations suggesting the choroidal nodules in choroidal Neurofibromatosis described by Viola $\mathrm{F}$ et al which also revealed that choroidal Neurofibromatosis is so common in NF-1 patients that can be proposed as a supplementary diagnostic criterion for NF-1 (Figure 2). Fundus examination and fluorescein angiography showed arterial macroaneurysm complicated by distal embolus. As various vascular and microvascular manifestations of NF-1 are being reported recently, further investigational studies for retinal vascular associations in NF-1 patients appears reasonable. Over and above, in every NF-1 patient, complete ophthalmic assessment including fundus examination seems essential to evaluate the choroid and the retina. This prevents potential sight- threatening complications of retinal vascular manifestation in such patients while it might help to make a stronger diagnosis of NF-1.

\section{Ethical Considerations}

\section{Compliance with ethical guidelines}

In this study, patient was anonymous; However, she was informed about the intended report.

\section{Funding}

This research did not receive any specific grant from funding agencies in the public, commercial, or not-forprofit sectors. Images were taken for free.

\section{Authors contributions}

Conceptualization: Meisam Ghanbari; Methodology: Mehrnoosh Maalhagh; Writing: Meisam Ghanbari; and Review and editing: Vahid Beigi.

\section{Conflict of interest}

The authors declared no conflict of interest. 


\section{References}

[1] Faro SH, Higginson SN, Koenigsberg R. Phakomatoses. Part I: Neurofibromatosis type 1: Common and uncommon neuroimaging findings. Journal of Neuroimaging. 2000; 10(3):138-16. [PMID]

[2] Hughes RA. The neurofibromatoses: A pathogenetic and clinical overview. London: Chapman and Hall Medical; 1994.

[3] Sippel KC. Ocular findings in Neurofibromatosis type 1. International Ophthalmology Clinics. 2001; 41(1):25-40. [PMID]

[4] Abdolrahmizadeh S, Plateroti AM, Recupero SM, Lambiase A. An update on the ophthalmologic features in phakomatoses. Journal of Ophthalmology. 2016; 2016:3043026. [DOI:10.1155/2016/3043026] [PMID] [PMCID]

[5] Viola F, Villani E, Natacci F, Selicorni A, Melloni G, Vezzola $\mathrm{D}$ et al. Choroidal abnormalities detected by near-infrared reflectance imaging as a new diagnostic criterion for Neurofibromatosis 1. Ophthalmology. 2012; 119(2):369-75. [DOI:10.1016/j.ophtha.2011.07.046] [PMID]

[6] Saylor WR, Saylor DC. The vascular leisons of Neurofibromatosis. Angiology. 1974; 25(8):510-9. [DOI:10.1177/000 331977402500803] [PMID]

[7] Moadel K, Yannuzi LA, Ho AC, Ursekar A. Retinal vascular occlusive disease in a child with Neurofibromatosis. JAMA Ophthalmology. 1994; 112(8):1021-3. [PMID]

[8] Thölen AM, Messmer EP, Landau K. Peripheral retinal and vascular occlusive disorder in a young patient with Neurofibromatosis 1. Retina. 1998; 18(2):184-6. [PMID]

[9] Mori F, Kawai M, Sato E, Igarishi S,Hikichi T,Yoshida A. Branch retinal vein occlusion in a Japanese patient with Neurofibromatosis 1. Japanese Journal of Ophthalmology. 2001; 45(6):634-5. [PMID]

[10] Muci-Mendoza R, Ramella M, Fuenmayor-Rivera D. Corkscrew retinal vessels in Neurofibromatosis type 1: Report of 12 cases. British Journal of Ophthalmology. 2002; 86(3):282-4.

[11] Lecleire-Collet A, Cohen SY, Vignal C, Gaudric A, Quentel G. Retinal ischemia in type 1 Neurofibromatosis. British Journal of Ophthalmology. 2006; 90(1):117. [DOI:10.1136/ bjo.2005.077701] [PMID] [PMCID]

[12] Abdolrahimzadeh S, Felli L, Piraino DC, Mollo R, Calvieri S, Recupero SM. Retinal microvascular abnormalities overlying choroidal nodules in Neurofibromatosis type 1. BMC Ophthalmology. 2014; 14:146. [DOI:10.1186/1471-2415-14146] [PMID] [PMCID] 